\title{
Auditoría externa en una ESE. Un ejercicio de apoyo en un sistema de costos
}

\section{External audit in an ESE. A support exercise in a cost system}

\author{
Eliecer Caraballo Hernández ${ }^{1}$
}

En Colombia en los últimos seis lustros se han dado importantes transformaciones en la organización del sistema nacional de salud, especialmente en relación con la calidad de la atención, convirtiéndola en uno de los pilares fundamentales de la prestación de los servicios de salud; precisamente, fue la Ley 10 de 1990 quien estableció la salud como un servicio esencial y obligatorio que podría ser prestado directamente a través del estado o mediante particulares, y fue así como determinó en sus articulado, que debía "establecerse un sistema de fijación de normas de calidad de los servicios de salud y los mecanismos para controlar y vigilar su cumplimiento"(1).

La trascendencia de la ley mencionada anteriormente se refleja y ratifica en el Artículo 48 de la Constitución Política de Colombia de 1991, donde se establece que la seguridad social y en especial, "la atención en salud es un servicio público obligatorio con cargo al estado, el cual se encargará de dirigir, orientar, regular, vigilar y controlar la prestación de los servicios públicos esenciales"; y se complementa el accionar de la Constitución Política en el Artículo 49 que instituye que, "Ios servicios de salud se organizarán de forma centralizada, con niveles de atención y con la participación de la comunidad. Se basará en principios de eficiencia, universalidad y solidaridad" (2).

Precisamente, en la Ley 100 de 1993, en el Artículo 225, se estatuye que las Empresa Promotoras de Salud-EPS cualquiera sea su naturaleza, deberán establecer sistemas de costos, facturación y publicidad. Los sistemas de facturación deberán permitir conocer al usuario por medio de una factura u otro mecanismo, los servicios y los correspondientes costos, discriminando la cuantía subsidiara por el Sistema General de Seguridad Social en Salud"(3). Asimismo, el parágrafo del Artículo 185 de la ley en mención establece que toda IPS contará con un sistema contable que le permita registrar los costos de los servicios ofrecidos.

Por su parte, en el Decreto 1281 de 2002 (4), se expiden las normas que regulan los flujos de caja y la utilización oportuna y eficiente de los recursos del sector salud y su utilización en la prestación del servicio de salud; basándose en los principios de eficiencia para la mejor utilización social y económica de los recursos financieros disponibles para que los beneficios que se garantizan con los recursos del Sector

${ }^{1}$ Mtr. en Administración de Empresas. Doc.Catedrático Universidad de Córdoba. eliecercaraballohernandez@gmail.com

Citación (Vancouver): Auditoría externa en una ESE. Un ejercicio de apoyo en un sistema de costos. :36-40 . doi: $\underline{10.21897 / 25394622.2568}$ 
Salud y se presten en forma adecuada y oportuna, y de oportunidad, que hace referencia a los términos dentro de los cuales cada una de las entidades, instituciones y personas, que intervienen en la generación, el recaudo [...] y que se garantice el acceso y la prestación efectiva de los servicios de salud a la población del país.

De la misma manera la Ley 1122 de 2007 en su Artículo 13, plantea que, las Entidades Promotoras de Salud - EPS de ambos regímenes, pagarán los servicios a los Prestadores de Servicios de salud habilitados, mes anticipado en un $100 \%$ si los contratos son por capitación. Si fuesen por otra modalidad, como pago por evento, global prospectivo o grupo diagnóstico se hará como mínimo un pago anticipado del $50 \%$ del valor de la factura, dentro de los cinco días posteriores a su presentación (5).

En general deben establecerse por parte del gobierno las medidas necesarias para asegurar el flujo ágil y efectivo de los recursos del Sistema, utilizando de ser necesario, el giro directo y la sanción a aquellos actores que no aceleren el flujo de los recursos. En este punto la normatividad vigente en el sector salud ha ido complementando la forma de asegurar el flujo de los recursos del sistema, creando mecanismos de control y vigilancia a través de los entes reguladores del gobierno nacional, estableciendo que las responsabilidades de las partes además de garantizar una prestación de servicio con calidad, también es la de dar información financiera veraz y oportuna conforme a los tiempos y estructuras definidas por normas como la Resolución 1121 de 2013, y la Circular 030 de $2013(6,7)$.

No obstante, el conflicto financiero dentro de una IPS pública o privada, se evidencia en la manera en el que se realizan los procesos y actividades en la distribución de recursos humanos, materiales y económicos, que buscan mediante la atención, la estabilización de pacientes en estado crítico y disminuir sus secuelas o complicaciones posteriores; y que se convierte en un descalabro para la mayoría de instituciones en muchas ocasiones por diferentes causales, destacando entre muchas el desconocimiento de la norma vigente, la debilidad en el levantamiento de procesos, procedimientos y flujos de información, y la resistencia al cambio por el personal asistencial y administrativo.

Sin embargo, dentro del Sistema General de Seguridad Social en Salud- SGSSS y cimentándose en el nuevo modelo de prestación de servicios que cambia el concepto de subsidio por el de oferta a la demanda, las IPS, como cualquier empresa productora de bienes y servicios, debe garantizar su autosuficiencia y subsistencia, por medio de la producción y venta de servicios asistenciales por un monto igual o superior a los gastos (3).

Teniendo en cuenta la normatividad vigente, expuesta en líneas anteriores, dentro del entorno actual de seguridad social, se deduce que la calidad en la atención es requisito fundamental de la prestación de los servicios de salud en el país, y que además es necesario desarrollar y estandarizar procesos dentro de todas las áreas de las entidades, que puedan Ilagar a garantizar ingresos que de una u otra forma fortalezcan los mecanismos usados en la sostenibilidad, el crecimiento y el desarrollo de las Instituciones Prestadoras de Servicios- IPS, y que además, sean fácilmente auditables para el mejoramiento de la calidad en salud, para incidir en las fallas y tomar correctivos oportunamente.

Dada las características del contador público en el salvaguardar y propender por el ordenamiento de los recursos públicos, una de sus funciones esenciales consiste en realizar acciones de auditorías señaladas dentro del sistema de salud colombiano, a los diferentes procesos y procedimientos asistenciales que se cumplen en los distintos servicios habilitados, para este caso en una Empresa Social del Estado -ESEy desde luego tratar de identificar los procesos y procedimientos administrativos de una IPS y proponer soluciones correctivas que contribuyan al mejoramiento del flujo de información necesaria, especialmente en la parte financiera. 
Cundo se desarrolla este tipo de auditoría, por supuesto que se examina sistemática e integralmente el grado de eficiencia en la aplicación de los procesos administrativos y asistenciales en las distintas unidades funcionales de la entidad y la manera en que su eficiencia influye en la efectividad de los procesos. Los hallazgos de la auditoría se deben describir conforme a los aspectos positivos, los cuales hacen referencia a las actividades, situaciones, acciones que se vienen desarrollando y que generan valor en la gestión del proceso o en la atención de los usuarios. En cuanto al riesgo, se refieren a la situación que representa un potencial incumplimiento a uno o varios requisitos de calidad, es decir, se manifiestan como una debilidad dentro del proceso y puede convertirse en una no conformidad. Frente a la No Conformidad, de igual manera se debe indicar el incumplimiento frente a uno o varios de los requisitos de calidad del sistema de gestión y en cuanto a la recomendación se opta por proponer que el equipo auditor, e incluso éste de manera individual dirija sus esfuerzos y capacidades hacia mejoramiento de uno o los aspectos encontrados durante el ejercicio de la auditoría.

En cuanto al trabajo de campo o la aplicación del ejercicio de la auditoría, es probable que en el acápite de gestión y análisis de información institucional, se encuentren hallazgos que naturalmente involucren lo relacionado con la habilitación de los servicios y corresponde al auditor, cargo que puede ser ejercido por un profesional con formación en el área financiera; desde luego que en este caso tendría que verificar el proceso de habilitación de todos los servicios intrahospitalarios y naturalmente que los ambulatorios, que cumplan con la normatividad vigente.

El proceso de auditoría es duradero y contribuye a través de algunos indicadores a un tipo de evaluación continua (8). El componente de la auditoría especialmente en el sector salud requiere del establecimiento de matices conceptuales precisos de manera tal, que es impreciso confundir terminológica y operativamente de eficacia, efectividad, eficiencia y equidad que correlacionan diferentes magnitudes. Frente a tal disyuntiva narrativa se debe precisar que la eficacia supone hacer las cosas, correlacionando la capacidad de una medida sanitaria con el logro de determinado efecto; siendo la efectividad la relación entre los objetivos previstos y los alcanzados. La eficiencia se adscribe al cumplimiento de los objetivos minimizando el empleo de recursos. La equidad se enmarca en la justicia social y busca con estándares mínimos lograr igualdad de oportunidades (9).

Es pues, inconcebible que una institución especialmente del sector salud o de índole particular, denomínese profesional independiente, gerente, administrativo, financiero, facturador, auditor, contratante o contratista, que no tenga claro unos procesos tan importantes como lo son la facturación, las glosas y las cuentas en general por venta de servicios de salud de una institución prestadora de éstos (10).

Si bien, desde el punto de vista económico, se considera que la situación de salud en el mundo es crítica por la multiplicidad de factores que intervienen en ella, se deben establecer políticas públicas en ésta materia que mínimamente, propendan por alcanzar algunos niveles pequeños de equilibrio por lo menos en lo que respecta a la prestación de los servicios de salud (11). Es aquí donde el profesional en finanzas o quien ejerza las veces de auditor alcance a desempeñar un papel relevante en los procesos públicos de control en el manejo adecuado de los recursos, lo que puede llegar a repercutir de manera óptima o acertada en la organización y su subsistencia.

En síntesis, para y durante el desarrollo de un proceso de auditoría externa y el establecimiento de un sistema de costos en cualquier entidad del sector salud independientemente del nivel de complejidad es importante el apoyo de la Dirección en cuanto a asignación de recursos, humanos, tecnológicos y físicos para todos los procesos permitan el logro de los objetivos institucionales. De la misma forma, se requiere de una dinámica de mejoramiento continuo en la institución, para lo cual es fundamental que 
desde ese direccionamiento se creen mecanismos de control y evaluación de los procesos que permitan fortalecer los aspectos críticos que se puedan evidenciar en el desarrollo de la auditoría como un proceso de atención y análisis documental (12), que por lo general, y de acuerdo al nivel de atención generará una propuestas de intervención conforme a la magnitud de los eventos o situaciones encontradas.

Probablemente en el proceso de auditoría se encuentren debilidades frente al desconocimiento de la estructura de los procesos, el uso y manejo inadecuado de la documentación y la información, las prácticas indebidas evidenciadas, así como la incorrecta aplicación de la normatividad vigente en los procedimientos auditados, requiere, dada la complejidad de los procesos que se deben auditar y la multiplicidad de factores que pueden Ilegar a afectar la calidad de la atención (13) por lo tanto, el fortalecimiento inmediato de acciones de sensibilización y capacitación. Originándose con esto, una urgente realización de un plan de mejoramiento continuo y fortalecimiento de un modelo empresarial competitivo en especial financieramente sostenible. El cual debe partir de la identificación de las variables identificadas y que ameritan ser intervenidas.

Otro elemento determinante en el proceso de una auditoría externa y su relación con el sistema de costos, está ligado a la implementación de un sistema integral y confiable de información en salud, a través del cual se logrará una ordenada y coherente estandarización de procesos. Dado que la información como elemento clave para la subsistencia de las instituciones y en especial las de carácter Público, no pueden darse el lujo, al contrario, deben incorporarse esfuerzos para que exista en la sociedad del conocimiento y la información, por aspectos ya demostrados y superados, cuando se elaboran por ejemplo manualmente los registros.

Por último, y no menos importante un aspecto abrumadoramente crítico y del cual no se debe sustraer ninguna administración gerencial y desde luego ninguna administración, está rela- cionado con la posibilidad de identificar la atención insegura o de presencia de malas prácticas en la prestación de los servicios de salud, por cuanto este es un pilar fundamental de la atención en salud, el cual también está sujeto a la supervisión permanente de su labor y de las implicaciones que pueden acarrear para la institución una inadecuada práctica de su función como entidad en el caso que nos ocupa, publica del sector salud. Desde luego, que estos hechos considerados en algunos casos por la normatividad como adversos (14), si logran demostrase su asociación con el estado de salud y condiciones de salud pueden generar inconvenientes, como es obvio a las finanzas y funcionamiento de la institución. 


\section{REFERENCIAS}

1. Hernández, Mario. Reforma sanitaria, equidad y derecho a la salud en Colombia. Cadernos de saúde pública 18 (2002): 9911001.

2. De Colombia, Constitución Política. Constitución política de Colombia. Bogotá, Colombia:1991.

3. Franco Giraldo, Álvaro. La ley 100 de 1993, la salud pública y la seguridad social en Colombia, 1995.

4. González Torres, Rafael Humberto. Identificación de fallas y presentación de oportunidades de mejora por devoluciones en la IPS Sociedad Clínica de Boyacá limitada año 2016.

5. Restrepo Villa, Román. A propósito de la Ley 1122 del 2007 Reforma del Sistema General de Seguridad Social en Salud en Colombia: esperanza, mito y realidad." Revista Facultad Nacional de Salud Pública 25.1 (2007): 90-105.

$6 . \quad$ Torres, Gloria Inés Valbuena, and Yolanda Cuellar Medina. Información financiera y costos utilizados para el sector salud en el municipio de Florencia. FACCEA-Revista Facultad de Ciencias Contables, Económicas y Administrativas 4.2 (2014): 122-130.

7. Sierra, Yessica Alexandra Beltrán, Irma Janeth Garzón Cano, and Pedro Nel Valbuena Hernández. Estimación del indicador de prestación del servicio de salud en Colombia (2007-2010); una base para la discusión sobre calidad de vida. Cuadernos Latinoamericanos de Administración 9.16 (2013): 79-99. 8. Heredia, Francisco Álvarez. Calidad y auditoría en salud. ECOE ediciones, 2015.

9. PISCOYA, José. Calidad de la Atención en Salud a través de la Auditoría Médica. En Anales de la Facultad de Medicina. Universidad Nacional Mayor de San Marcos, 2000. p. $227-240$

10. Martínez, Mauricio Leuro, and Irsa Tatiana Oviedo Salcedo. Facturación y auditoría de cuentas en salud. Ecoe Ediciones, 2016

11. Rojas OF. Determinantes sociales de la salud y la acción política. Rev Hum Med. 2013; 13(2): 279-291
12. Naranjo Álvarez, Yésica, and Daniela Jiménez Ramírez. Auditoría externa con enfoque en ISO 9001 versión 2015, Servicios de Salud San Vicente Fundación, 2021-1.

13. Infante Takey, Henry Ernesto. Un modelo para determinar los factores que influyen en la mejora de procesos en la atención de pacientes en los centros de salud a través de Excelencia Empresarial, BPM y Reingeniería de Procesos. 2013.

14. Osorio Martínez, David Alejandro, et al. Indicios de Atención Insegura durante los traslados internos desde el servicio de urgencias a otros servicios; 2020. 